\title{
INDIVIDUALLY-FITTED CORNEAL LENSES MADE TO CORNEAL MOULDS*
}

\author{
BY \\ J. DALLOS \\ London
}

THE practical performance of a contact lens, haptic or corneal, is a function of its physiological effect. The latter is the composite outcome of optical, biochemical, and biophysical properties, of which the optical properties are under such technical control as to exceed in precision any practical requirement. Regarding biochemical desiderata, the guiding principle of non-interference is achieved by the maintenance of osmotic balance through unhampered precorneal fluid exchange, which is at its optimum in haptic lenses when a flush-fitting is carried out to anatomical standard (i.e. accurate surface-to-surface co-adaptation), then eased over limbal and marginal transitions, and, finally, effectively ventilated. In the biophysical field, flush-fitting assures minimal local interference with conjunctival physiology, $\dagger$ and the bridging of the two transitional zones assures a uniform smoothness of tangential displacement of the perforated lens during eye movements. Dynamic irritation is then at its minimum and the precorneal capillary distance is securely maintained at constant level. Tear circulation at its optimum will maintain osmotic constancy.

The principle of least interference applies no less to corneal lenses but, whereas in my experience technical tolerance of accuracy in conjunctival haptics is in the $50 \mu$ range descending to $25 \mu$ only over the corneal area, in corneal fitting the tolerance descends further to a single figure in microns, a different order of magnitude altogether.

The great advantage of corneal lenses over haptic lenses is their potentially total non-interference with the bulbar conjunctiva, avoiding that protective mucous discharge to which some conjunctivae are provoked whilst dynamic irritation is otherwise subliminal. This may become a major nuisance in the practical use of haptic lenses. The tarsal, limbal, and palpebro-marginal conjunctiva, however, are still involved. Limbal oedema may show within minutes, and will invariably appear if any corneal lens is forced by the lid movements beyond the upper corneal limbus. The lens soon becomes embedded in the swollen limbal conjunctiva; this immobilization produces precorneal stagnation with the usual osmotic sequelae. The rest of the

\footnotetext{
* Received for publication December 12, 1963

+ Any deviation from uniform anatomical co-adaptation (flush-fitting) creates comparatively tight and loose areas at the same time. Looseness, quite generally, invites air bubbles which churn into froth and will inhibit tear circulation in the manner of capillary air embolism. In the corneal region this causes, apart from optical interference, epithelial after-effects. Loose peripheral sectors, particularly mobile margins, cause dynamic irritation.

Tightness over scleral areas interferes with vascular as well as with tear circulation, localized compression causing pain: firs discomfort and, if repeated before full recovery, intolerance. In the corneal region comparative tightness inhibits fluid exchange but as osmotic forces will still preponderate over molecular forces of adhesion, the epithelium will swell; its growing volume with its constant basal area demands an extension of its surface creating a mesh of wrinkles with pits and furrows in between, over which a collection of gas bubbles nestle, imitating froth. Clinically froth from looseness is completely and invariably prevented by traces of castor oil; froth from tightness remains unaffected. 
conjunctiva is troublesome only when the tarsomarginal pain threshold is low. It may be too low to overcome by corneal fitting and then becomes an indication in favour of haptic lenses.

Corneal consequences, both epithelial (oedema in various stages and clinical appearances from veiling to erosion) and interstitial (keratoxysis, warping), are frequent and much more difficult to avoid than with haptic lenses. The reason is that, if the contacting surfaces in the apical area are of identical curvature, and the lens large enough to maintain this contact, then the precorneal flow is at stagnation point. If on the other hand, in order to avoid this trouble, the lens is allowed to float, its peripheral zone will massage the apex or, if more stationary, will press the apex out of its optical precision and cause a distortion (spectacle blur). This distortion will last for anything from minutes to hours, and if over-night recovery is insufficient and is consistently ignored, it may continue for days or weeks.

Corneal fitting has to-day reached a stage analogous to Heine's multi-curve combinations in the Zeiss type of spherical sclero-corneal contact lenses. They are a marked improvement on single curve variations but suffer from a basic anatomical inaccuracy, inherent in the geometrical conception of their design.

As in haptic lenses, so in corneal lenses, no set of ready-made varieties, no choice in any combination of successive spherical or toric curves (specifications) will approach the physiological requirements of the individual so closely as can the application of individual anatomy in fitting and manufacture. It is remarkable that, although the discovery of corneal moulding was published before that of scleral moulding, it has hitherto been little used for corneal fitting, and quite neglected in the field of corneal lenses. This neglect is even more remarkable when one considers that the cornea is hard and that a cast of it and of its immediate surrounds can be made both precise and reliable by present-day methods and materials. Keratometer readings of a wet hydrocolloid cast are practically identical with those of the cornea from which the cast was taken.

Corneal lenses made from a hard mould of this cast were reported to be successful in some conical corneae too distorted to be fitted with any multicurve combination. Apart from these exceptionally misshapen corneae, where the most modest visual improvement is a major achievement, direct moulding was abandoned; for it has the essential disadvantage of requiring optical treatment of its inside as well as of its outside surface. And whereas the loss of $10 \mu$ of material in optical smoothing and polishing does not affect the fitting properties of a haptic lens unduly, it is outside the permissible error for a corneal lens.

Without optical treatment to its inside surface, although a moulded lens can be a closer anatomical approach than any other, it is rarely acceptable as a visual aid in preference to fully corrective glasses. Also, anatomical perfection would only be the ultimate aim in fitting if it coincided with the functional optimum, which it does no more in corneal than in haptic fittings. In order to preserve osmotic balance, constant tangential sliding is as necessary as in haptic lenses, although the use of optically inactive $(200 \mu$ diameter) micro-perforations can occasionally reduce this requirement. The aim, therefore, is not so much the precise anatomical reproduction of the corneal surface, including its toric-paraboloid periphery, as the production of the smoothest functional co-adaptation between lens and the individual cornea. 
If this requirement is to be met in a corneal lens, it would seem that a functional method of production must be employed rather than the usual static reproduction obtained by moulding or plastic processing. Technical machinery that causes the lens-in-making to move over a solid replica of the individual cornea, imitating the floating of the corneal lens from limbus to limbus, could produce a fitting surface of smoothest functional co-adaptation. Machinery employing such a movement has long existed for making toric curvatures by smoothing a preformed, approximately curved, lens to a static hard toric surface; it is used in the manufacture of cylindrical haptic lenses for the correction of lenticular astigmatism. The adaptation of machinery, methods, and materials to produce the desired results is past the experimental stage, and the correlation of these with the results obtained by the methods hitherto available is now in progress.

The proposed new technique is not expected to be the last word in contact lens fitting. Indeed, no single type of fitting is ever likely to cover all requirements. Thus, a low corneal or tarsomarginal pain threshold still demands a haptic fitting. And an occasional case still requires a firm limbal fit for optical reasons (some uniocular aphakics and most wearers of stenopaeic contact lenses are intolerant of the slightest tangential displacement of their contact lenses during eye movements) or for haptical reasons (an eccentric terraced corneal graft or a very steep conical cornea will not always hold a corneal lens, and if it is fitted with a ventilated haptic lens, an air bubble will appear in the pupillary area). Steep conical corneae call for unperforated haptic fitting, whereas corneae with a long history of keratitis and aphakic eyes with their diminished rate of intra-ocular metabolism and reduced limbal sensitivity are best suited by a full-sized corneal cap with circular limbal support.

Subjective performance in haptic lenses, including these apparent exceptions, reaches an optimum when they comply with the general principle of physiological non-interference. The means to obtain this is exact objective fitting. In corneal lenses, the nearest approach to this principle is a lens with the smoothest functional co-adaptation. A standardized technique, using the individual corneal mould to create a functionally co-adaptive surface, and thereby automatically eliminating fitting errors, is now being developed. It is hoped that this method will result in an improvement over present-day standards of performance in corneal lenses.

I should like to thank Mr. F. A. Williamson-Noble and Dr. Paul Wingate for their great kindness in helping me with the preparation of this paper. 